\title{
Psychogenic non-epileptic seizures: management and prognosis
}

Kate Irwin, Melinda Edwards, Richard Robinson

\begin{abstract}
Aim-To determine the outcome and identify predictive factors in children with psychogenic non-epileptic seizures (PNES).

Method-The biographies of 35 children with PNES, attending a tertiary paediatric neurology centre, were reviewed.

Results-Thirty five children attending the department between 1987 and 1997 were evaluated at a mean follow up of 4.6 years. The age range was 6-18 years. Twenty four were girls and 11 were boys. Eleven patients had a diagnosis of epilepsy with PNES, the remainder having PNES alone. Cause fell into four categories: a history of violence, abuse, or neglect; a high level of anxiety; dysfunctional family relationships; and attention seeking or avoidance behaviour. Management in all but five cases involved assessment and follow up by a child psychologist or child psychiatrist. The outcome was encouraging, with $66 \%$ of patients becoming PNES free. A further $23 \%$ have $>50 \%$ reduction in the frequency of PNES. Only two have had no reduction. Outcome was best in the group without epilepsy.

Conclusion-These results suggest that the prognosis of PNES is better in children than in adults, perhaps because causes are more likely to be external to the child, more easily identified, and more amenable to prompt intervention. The importance of good assessment, good communication, and a treatment plan that includes both symptom management and addressing the precipitating and perpetuating factors is emphasised.

(Arch Dis Child 2000;82:474-478)
\end{abstract}

Keywords: psychogenic seizures; pseudoseizures; non-epileptic seizures; epilepsy

There is no entirely satisfactory term for the phenomenon we are discussing. ${ }^{2}$ "Nonepileptic seizures" and "pseudoepileptic seizures" logically should include seizures secondary to cardiac syncope and other causes, just as "psychogenic seizures" should include epileptic seizures triggered by reading, anxiety, and other cerebral events. "Pseudoseizures" attempts to deny the validity of the events altogether. We have adopted the term "psychogenic non-epileptic seizures" (PNES) to describe a type of episodic behaviour that mimics epileptic seizures but is to be distinguished from them.
The diagnosis of PNES can be difficult in epilepsy practice, particularly when the patient also has epilepsy. The advent of videotelemetry has reduced the diagnostic pitfalls $\mathrm{s}^{3-6}$ and the need for prolactin measurements, ${ }^{78}$ which can be inconclusive.

There is substantial literature on PNES in adults. A proportion of adults with PNES have a history of both physical and sexual abuse; others have complex personality disorders, alcoholism, anxiety, or depression. ${ }^{9-11}$ The prognosis in adults can be poor, although recent literature suggests a promising response to psychotherapy. ${ }^{12}$

Less has been published on this condition in children, although it has been suggested that the prognosis is better in children and adolescents than in adults. ${ }^{13}$ The aim of this study was to gain a better understanding of the causes, management, and outcome of this complex condition in the paediatric age group. The study is uncontrolled, retrospective, and descriptive. Conclusions about the effectiveness of intervention are necessarily anecdotal but nevertheless often compelling.

\section{Patients and methods}

The case notes of all patients attending the paediatric department between 1987 and 1997 who had a diagnosis of PNES were reviewed. The patients were divided into those with concomitant epilepsy and those without. Other causes of paroxysmal events had been excluded by relevant investigations including blood glucose levels and electrocardiogram (ECG). When the diagnosis of epilepsy coexisted with PNES, the diagnosis of epilepsy was made by observation of an event and/or by electroencephalographic (EEG) recording of an attack. The diagnosis of PNES was made by history and/or observation of the episode and, in some cases, videotelemetry. Differentiating a nonepileptic seizure can be difficult even for the most experienced observer. Methods employed to aid the diagnosis included attempted opening of the eyelids to assess resistance in someone in apparent deep coma; similarly obtaining a blink reflex on tickling the eyelashes, and allowing raised arms to fall (they never fall twice across the face during a PNES). Incontinence is rare and self injury, at most, minor. Accidental injuries can occur during PNES and therefore did not rule out this diagnosis.

Specific information sought included a description of the seizure and the likely source of the model for this seizure. Difficulties at home and at school were noted, as well as problematic family characteristics and second- 
ary gains. Other psychosomatic illness in the child or family was recorded.

Outcome measures included seizure frequency, school attendance, social adaptation, and the frequency and severity of somatising behaviour. Seizure freedom was considered to have been achieved if the patient had not experienced any episodes for at least six months before this review.

The relation between cause and outcome was critically reviewed. The level of significance of differences was assessed using the $\chi^{2}$ test.

\section{Results}

Thirty five patients were evaluated at a mean follow up of 4.6 years. The age range of the patients was $6-18$ years, the mean age of presentation being 14.1 years. Thirty of the patients presented at age 11 or over. Twenty four patients were girls and 11 were boys. Three children had moderate learning difficulties.

The diagnosis of PNES was made by the history alone from witnesses in 18 cases, from the history and clinical observation of a seizure in four cases, and by history and videotelemetry in the remaining 13 (table 1).

Eleven subjects had tonic clonic PNES, 10 had prolonged blank spells during which they stared straight ahead unresponsively, 10 had episodes during which they slid to the floor and remained still and unresponsive ("swoons"), and four described a mixture of events (table 2). There was no clear pattern between PNES type and age of the patient.

Eleven children had a primary diagnosis of epilepsy. Only three children in this group exhibited prolonged blank spells or swoons compared with 17 of the 24 without epilepsy $(\mathrm{p}<0.05)$. Seven modelled their non-epileptic events on their epileptic seizures, which included tonic clonic, absence, and complex partial seizures. In the remainder no one seizure type predominated. Causes were identified in all patients in the group with epilepsy which, in the main, arose from the primary diagnosis itself. Secondary gains were apparent, with school avoidance in six cases (because of bullying in three and falling behind in schoolwork in three) and attention seeking in three. One child was frightened by his auras and had non-epileptic sequelae which appeared effective in gaining parental attention

Table 1 Diagnostic methods for the group as a whole

\begin{tabular}{lc}
\hline Diagnostic method & $\begin{array}{c}\text { Number } \\
(n=35)\end{array}$ \\
\hline History alone & 18 \\
History and seizure observation & 4 \\
History, seizure observation, video EEG & 13 \\
\hline
\end{tabular}

Table 2 Seizure description in the group as a whole

\begin{tabular}{ll}
\hline Seizure description & $\begin{array}{l}\text { Number } \\
(n=35)\end{array}$ \\
\hline Tonic clonic type & 11 \\
Prolonged blank spells & 10 \\
Black outs & 10 \\
Other & 4 \\
\hline
\end{tabular}

Table 3 Causes of psychogenic non-epileptic seizures in the group with epilepsy

\begin{tabular}{ll}
\hline Cause & $\begin{array}{l}\text { Number } \\
(n=11)\end{array}$ \\
\hline Attention seeking & 3 \\
School avoidance & \\
$\quad$ Bullying & 3 \\
$\quad$ Poor performance & 3 \\
Anxiety & 1 \\
Family stress & 1 \\
\hline
\end{tabular}

Table 4 Types of seizures in group without epilepsy

\begin{tabular}{ll}
\hline Seizure description & $\begin{array}{l}\text { Number } \\
(n=24)\end{array}$ \\
\hline Swoons & 9 \\
Prolonged blank spells & 8 \\
Clonic movements with pelvic thrusting & 5 \\
Other & 2
\end{tabular}

Table 5 Causes of psychogenic non-epileptic seizures in the group without epilepsy

\begin{tabular}{ll}
\hline Cause & $\begin{array}{l}\text { Number } \\
(n=24)\end{array}$ \\
\hline History violence/abuse & 8 \\
Domestic stress & 4 \\
School avoidance & 4 \\
Maternal overdependence & 3 \\
Unknown & 5
\end{tabular}

and reassurance. One adolescent found his tense and angry family difficult to manage (table 3).

Although two of the 24 children without epilepsy knew a close family member with epilepsy, neither they nor the others had a seizure model. Nine had swoons during which they lay still and unresponsive, eight had prolonged blank spells involving long periods of staring straight ahead, five had tonic clonic movements with pelvic thrusting, and two had a mixture of events (table 4 ).

A likely cause was identified in 19 of the 24 . Eight had traumatic histories of violence and physical and sexual abuse. Four were subjected to significant domestic tension, for example one girl was caught up in an emotional battle between separated family members. Four were avoiding school because of bullying or poor performance. Three subjects had dysfunctional relationships with their mothers (because of overidentification and overinvolvement), and, in all, the episodes disappeared during enforced separation from their mother by hospital admission. In the remaining five children, no clear precipitant was identified, although the attention gained from their PNES seemed to perpetuate the problem (table 5).

Management in most cases included early referral to a child psychologist. All children with a primary diagnosis of epilepsy were also reviewed regularly by a paediatric neurologist. Once the diagnosis of PNES was clear in the group without epilepsy, and had been accepted by the patient and family, the patient was discharged from the care of the neurologist to avoid the appearance of inconsistency. In some cases, the diagnosis and management required admission to hospital, for example in all children who had an abnormal maternal relationship. Two of the eight cases with a history of abuse or violence were referred for pro- 
longed management by a child psychiatrist. Three of the 35 patients required a three month stay in a psychiatric unit (table 6).

Outcome varied considerably between groups. The children with epilepsy had the worst outcome. Only six of these (54\%) were PNES free compared with $71 \%$ in the group without epilepsy $(p>0.05)$. The three children who were avoiding school because of bullying were moved to different schools, and the PNES ceased. The three children who had academic difficulties improved when they received extra help; one of these continues to have episodes when she is exceptionally stressed. Reassurance, education about epilepsy, and coping strategies have helped the boy frightened by his auras, although he continues to have occasional PNES. The adolescent suffering domestic stress continues to have PNES, with the family refusing to engage with any mental health services. Two cases in this group have not shown any improvement in the frequency of their events. One of these has moderate learning difficulties and has not been reviewed by a child psychologist. Another has not attended school for 15 months and is now displaying unmanageable behaviour. She failed to engage with the child mental health services. Good control of this child's epilepsy did not affect the frequency of PNES (table 7).

The 24 cases without an initial diagnosis of epilepsy have had a better outcome. Seventeen $(71 \%)$ are now seizure free, with a further four having $>50 \%$ reduction in their episodes. One of the children who is seizure free now has regular attacks of hyperventilation, and another has stopped having seizures but continues to suffer considerable problems with social relationships. The five whose PNES were perpetuated by the attention gained by them responded to psychological intervention, but two continue to have episodes at times of stress or upset in the family or at school. They required a mean of six months follow up with a child psychologist. The six with domestic stresses improved when the problem was tackled with the whole family, after a mean of four months of psychological input. Two of these patients have grown up and left home; their outcome is not known. The four who were avoiding school responded to changing school, finishing school, and management of bullying. The six with a history of physical and sexual abuse,

Table 6 Follow up of group as a whole

\begin{tabular}{lc}
\hline Management & $\begin{array}{c}\text { Number } \\
(n=35)\end{array}$ \\
\hline Paediatric neurology team alone & 5 \\
Neurology team and child psychologist & 6 \\
Child psychologist alone & 20 \\
Psychologist and child psychiatrist & 4 \\
\hline
\end{tabular}

Table 7 Outcome in the group with epilepsy

\begin{tabular}{ll}
\hline Outcome & $\begin{array}{l}\text { Number } \\
(n=11)\end{array}$ \\
\hline PNES free & 6 \\
$>50 \%$ reduction & 3 \\
No improvement & 2 \\
\hline
\end{tabular}

PNES, psychogenic non-epileptic seizures.
Table 8 Outcome in the group without epilepsy

\begin{tabular}{ll}
\hline Outcome & Number $(n=24)$ \\
\hline Seizure free & 17 \\
$>50 \%$ reduction in PNES & 4 \\
No improvement & 1 \\
Unknown & 2 \\
\hline
\end{tabular}

PNES, psychogenic non-epileptic seizures.

violence, and neglect required regular sessions with a child psychologist over a long period of time. Four were discharged, episode free, after a mean of 18 months follow up. In all of these, the trauma of their past had been explored. One continues to have occasional episodes and remains under the care of a child psychologist. The neglect by her mother continues. One child continues to have prolonged and regular PNES; work with a psychologist is continuing and details of her traumatic past continue to emerge. All three cases in which the episodes were caused by overdependence of the child's mother required admission to hospital and periods of maternal separation. All of these improved when the mother also accepted psychological help; only one is currently PNES free (table 8).

\section{Discussion}

Although Williams et al, ${ }^{14}$ Finlayson et al, ${ }^{15}$ and Holmes et $a l^{4}$ highlighted the problem of PNES in children and adolescents in the late 1970s, few follow up data are available. ${ }^{316-18}$ Wyllie et $a l^{\beta}$ studied 18 children and adolescents, $78 \%$ of whom were PNES free after an average of 30 months. Details of the causes were scanty in this study. The same group of patients was compared with a similar group of adults with PNES. ${ }^{13}$ Significantly more of the children than the adults were free of PNES at the end of the study. Metrick et $a l^{17}$ reviewed 27 children with non-epileptic events. This study concentrated on different types of non-epileptic attacks which included physiological events and abnormal movements. They found that children misdiagnosed as having epilepsy were more likely to have physiological events than PNES. We do not discuss that group of children here. Again, details of causes of the PNES are scarce. Buchanan et $a l^{16}$ outlined the outcome of 50 patients with PNES. They did not differentiate between adults and children in their study, rather dividing the group into those with an acute history of PNES and those with a chronic history. A cause for the PNES was found in $94 \%$ of patients and included stress, attention seeking, illness behaviour, and abuse. The study reported a high incidence of personality disorders, depression, and anxiety in the chronic group. Not surprisingly, the acute group had a considerably better outcome.

Children with epilepsy only represent 31\% of our sample. Although it is probable that children with epilepsy are more likely to have PNES than those without, ${ }^{16} 171920$ absolute numbers would suggest PNES are more likely to be found in children without epilepsy. ${ }^{121}$ Few patients had a model for their seizures. Some $64 \%$ of those with epilepsy used their own epileptic seizures as a model; only $8 \%$ of 
those without epilepsy reported a personal knowledge of epilepsy. This contrasts with reviews of adult patients by Volow ${ }^{22}$ and Hopkins, ${ }^{23}$ who state that most people with PNES have experience of epilepsy.

Fewer children in this group were found to be "attention seekers" than suggested in other reports; $8.5 \%$ of the group as a whole compared with $33 \%$ of the 50 patients in the study by Buchanan et al..$^{16}$ It is important to differentiate between the precipitating and the perpetuating factors involved in each case. Behaviour can often be labelled in terms of continuing gains despite a lack of knowledge of the precipitating circumstances.

A likely explanation for the PNES emerged in most of our cases. An obvious secondary gain or predicament allowed management to be focused on the cause, leading to eventual resolution of the attacks. Abuse has often been implicated as a cause of PNES. ${ }^{24}{ }^{25}$ Eight of our children had a history of abuse. The outcome was excellent in most, possibly because the problem was detected sufficiently early to allow prompt intervention. These results compare favourably with those in adults who for years repress a history of early abuse. ${ }^{22}$ This confirms the explanation of Buchanan et $a l^{16}$ that poor prognosis is related to chronicity and complex psychological and psychiatric problems.

Young patients may require only relatively simple measures to lower stress levels, thereby reducing their attacks; for example, when the problem was clearly school related, the children who moved to new schools or were offered extra educational support improved rapidly. Domestic stress may decrease with support and certainly diminishes when the young person leaves home. Causes lying external to the child are more susceptible to recognition and hence intervention than predicaments arising in adults whose long history and entrenched maladaptive behaviour is difficult to modify.

PNES are a challenging problem for all concerned in the diagnosis, management, and care of the young patient. It is important for the diagnosis to be unambiguously communicated to families, including an explanation of the role of stress or emotional factors in the cause of seizures. The rationale for a treatment programme that focuses on psychological rather than medical issues should then be clear. It is important for the diagnosis to be conveyed positively, both with regard to the expectations of a good prognosis and recovery, and in terms of there being no shame or blame attached to a non-organic cause.

Prompt intervention from a child mental health professional is important to prevent families becoming entrenched in a medical approach. The initial focus is to contain and safely manage seizures while exploring any likely precipitating stresses and dealing with these. It is crucial for any management plan to be explicitly shared with and understood by families and all other professionals involved with the child, including school and community paediatric services. A letter outlining the nature of the attacks and the management plan, which is distributed to all concerned, can usefully facilitate consistency in management.

The aims of treatment are to help the family and child develop a greater understanding of the nature of PNES, to be able to control and contain the seizures safely, and to identify factors that are responsible for either triggering or maintaining the seizures. Initially a focus of "living safely" with the seizures can be helpful with normalising daily activities. This reduces the heightened emotional arousal and attention that is focused on them when seizures occur. Stress management techniques including relaxation therapy and cognitive approaches can be used. Past or current emotional stresses are explored and strategies identified which enable the child to feel better equipped to deal with the situation. This may include a focus on acquiring social skills, raising self esteem, and helping the child to confront or resolve issues with others, either in the family or outside. Direct interventions may involve dealing with the child's academic needs at school or putting in place programmes to deal with difficult peer relationships such as bullying. In more severe cases in which the child's safety is considered to be at risk or the agreed management plan does not contain the family's anxieties, it is more appropriate to admit the child to a psychiatric unit for more intensive exploration of issues and therapeutic help.

Our experience would suggest that the outcome is less favourable in patients with epilepsy, who are beset by problems arising from their primary diagnosis. Additional difficulties such as school avoidance or medical constraints create a complex predicament, which may involve exploring implications of the diagnosis of epilepsy for the child and the family. In turn, this may enable them to modify their own unhelpful responses and those of others.

This report is descriptive only. It does not seek to test hypotheses. The results are nevertheless encouraging. PNES in children have a good prognosis provided that the diagnosis is made early, a cause is identified and addressed, and there is appropriate psychological intervention. The diagnosis may be more common in children who do not have a primary diagnosis of epilepsy than in those who do.

1 Trimble M. Pseudoseizures. Neurol Clin 1986;4:531-48 2 Scull D. Pseudoseizures or PNES (NES); 15 synonyms [letter]. I Neurol Neurosurg Psychiatry 1997;62:200.

3 Wyllie E, Friedman D, Rothner D, et al. Psychogenic seizures in children and adolescents: outcome after diagnosis by ictal video and electroencephalographic recording. Pediatrics 1990;85:480-4

4 Holmes GL, Sackellares JC, McKieran J, et al. Evaluation of childhood pseudoseizures using EEG telemetry and childhood pseudoseizures using EEG telem
videotape monitoring. F Pediatr 1980;97:554-8.

5 Ives JR, Gloor P. A long-term time lapse video system to document the patients spontaneous clinical seizure synchronised with the EEG. Electroencephalogr Clin Neurophysiol 1978;45:412-16.

6 Boon PA, Williamson PD. The diagnosis of pseudoseizures. Clin Neurol Neurosurg 1993;95:1-8.

7 Wyllie E, Luders H, McMillan JP, et al. Serum prolactin levels after epileptic seizures. Neurology 1984;34:1601-4.

8 Trimble MR. Serum prolactin in epilepsy and hysteria. BMf $1978 ; 2: 1682$.

9 Stewart RS, Lovitt R, Stewart RM. Are hysterical seizures more than hysteria? Am $\mathcal{F}$ Psychiatry 1982;139:926-8.

10 Roy A. Hysterical fits previously diagnosed as epilepsy. Psychol Med 1977;7:271-3.

11 Vanderzant CW, Giordani B, Berent S, et al. Personality of patients with pseudoseizures. Neurology 1986;36:664-8. 
12 Aboukasm A, Mahr G, Gahry BR, et al. Retrospective analysis of the effects of psychotherapeutic interventions on outcomes of psychogenic nonepileptic seizures. Epilepsia

13 Wyllie E, Friedman D, Luders H, et al. Outcome of psychogenic seizures in children and adolescents compared with adults. Neurology 1991;41:742-4.

14 Williams DT, Spiegal H, Mostofsky DI, et al. Neurogenic and hysterical seizures in children and adolescents: differential diagnostic and therapeutic considerations. $A m \mathcal{F}$ Psychiatry 1978;135:82-6.

15 Finlayson RE, Lucas AR. Pseudoepileptic seizures in children and adolescents. Mayo Clin Proc 1979;54:83-7.

16 Buchanan N, Snars J. Pseudoseizures (non epileptic attack disorder): clinical management and outcome in 50 patients. Seizure 1993;2:141-6.

17 Metrick ME, Ritter FJ, Gates JR, et al. Nonepileptic events in childhood. Epilepsia 1991;32:322-8.
18 Lancman ME, Asconape JJ, Graves S, Gibson PA. $\mathcal{F}$ Child

Neurol 1994;9:404-7.
Trimble M. Pseudoproblems pseudoseizures. Br F Hosp Med 1983 Apr:326-33.

20 Krumholz A, Niedmeyer E. Psychogenic seizures: a clinical study with follow-up data. Neurology 1983;33:498-502.

21 Luther JS, McNamara JO, Carwile S, et al. Pseudoepileptic seizures: methods and video analysis to aid diagnosis. Ann Neurol 1982;12:458-62.

22 Volow MR. Pseudoseizures: an overview. South Med f 1986; 79:600-7.

23 Hopkins A. Pseudoseizures. QfM 1989;71:473-5.

24 Gumnut RJ, Gates JR. Psychogenic seizures. Epilepsia 1986; 27(suppl 2):S124-9.

25 Betts T, Boden S. Diagnosis, management and prognosis of a group of 128 patients with nonepileptic attack disorder. Part II. Previous childhood sexual abuse in the aetiology of these disorders. Seizure 1992;1:27-32.

\title{
Sneha-India and the International Council for Research into the Fetal Origins of Adult Disease
}

\section{First World Congress: Fetal Origins of Adult Disease}

\author{
Sponsored by the British Medical fournal \\ 2-4 February 2001, Mumbai, India
}

Poor fetal growth is associated with an increased risk of adult cardiovascular disease and diabetes, which has led to the hypothesis that these disorders originate through undernutrition in utero. Evidence also links fetal growth with later osteoporosis, neurological and psychiatric disease, hormone related cancers and atopy. This conference will bring together, for the first time, clinicians, epidemiologists, and basic scientists working in this field.

Topics include:

Cardiovascular disease

Diabetes

Cancer

Osteoporosis

Asthma

Ageing

Mental health

Maternal nutrition

Control of fetal growth

Hormonal programming

Immune function

Strategies for preventing disease

Plenary speakers include: Claude Lenfant (USA), Nick Hales (UK), Christopher Martyne (UK), Chittaranjan Yajnik (India), Michael Meaney (Canada), Jeffrey Robinson (Australia), Jane Harding (New Zealand), Kent Thornburg (USA), John Challis (Canada), Alan Jackson (UK), Keith Godfrey (UK), Patrick Bateson (UK), Peter Gluckman (New Zealand).

Scientific committee chairman: David Barker, Southampton, UK

Organising committee chairman: Anand Pandit, Pune, India

Further details from: Ms Alifiya S Motiwala (tel: +91 22651 6439/645 6763; fax: +91 22651 6438; email: mrcssc@vsnl.com).

Alternatively, fill in the "Yes, I am interested" reply slip on our website: www.sneha-india.org 